\title{
Fiber-optic sensor for liquid level measurement
}

\author{
J. E. Antonio-Lopez ${ }^{1,2}$ J. J. Sanchez-Mondragon, ${ }^{1}$ P. LiKamWa, ${ }^{2}$ and D. A. May-Arrioja ${ }^{3, *}$ \\ ${ }^{1}$ Photonics and Optical Physics Laboratory, Optics Department, Instituto Nacional de Astrofísica, \\ Óptica y Electrónica (INAOE), Apartado Postal 51 y 216, Tonantzintla, Puebla 7200, Mexico \\ ${ }^{2}$ CREOL, The College of Optics and Photonics, University of Central Florida, Orlando, Florida 32816-2700, USA \\ ${ }^{3}$ Departamento de Ingeniería Electrónica, UAM Reynosa Rodhe, Universidad Autónoma de Tamaulipas, \\ Carretera Reynosa-San Fernando S/N, Reynosa, Tamaulipas 88779, Mexico \\ *Corresponding author: darrioja@uat.edu.mx
}

Received June 3, 2011; revised August 4, 2011; accepted August 9, 2011; posted August 10, 2011 (Doc. ID 148671); published August 29, 2011

\begin{abstract}
A novel (to the best of our knowledge) liquid level sensor based on multimode interference (MMI) effects is proposed and demonstrated. By using a multimode fiber (MMF) without cladding, known as no-core fiber, liquids around the MMF modify the self-imaging properties of the MMI device and the liquid level can be detected. We show that the sensor exhibits a highly linear response with the sensing range and multiplexed operations easily controlled by just modifying the length of the no-core fiber. At the same time, we can measure the refractive index of the liquid based on the maximum peak wavelength shift. We can also use the sensor for continuous and discrete liquid level sensing applications, thus providing a liquid level sensor that is inexpensive with a very simple fabrication process. (C) 2011 Optical Society of America
\end{abstract}

OCIS codes: $\quad 060.2310,060.2370,280.4788,120.0280$.

Liquid level sensing is very important in many applications in which monitoring of the liquid's volume is required. This is particularly useful for flammable hydrocarbons or fuels and of chemicals in industrial and processing plants. Over the years, a wide range of liquid level sensing techniques based on mechanical, electrical, and optical methods has been reported [1-5]. Electrical liquid level sensors are widely employed, but their application is limited if the liquid to be monitored is conductive or corrosive or if the environment is potentially explosive. Optical fibers offer several advantages as compared to other technologies, and they have been the subject of intense research for several years [6]. Some of the key advantages include low power consumption, ability to withstand corrosive environments, immunity from electromagnetic interference, large distance between signal generation/detection, and high sensitivity. Therefore, liquid level sensing of flammable fluids or explosive environments are well suited applications for optical fiber sensors, because they do not require electrical signals. In general, for liquid level measurement we have two types of level sensor: continuous measurement [6-10] and discrete or point measurement [11-13]. Discrete sensors are designed to detect the presence of liquids at a particular point by using exposed fiber gratings and special fiber tips, which rely on intensity variations as the liquid level makes contact with the sensing region. Continuous monitoring of the liquid level typically employs evanescent wave interaction by using long-period fiber gratings, bent side-polished plastic optical fiber, or etched D-shaped optical fiber. In all cases, they need to use a special fiber structure, a special fiber treatment, or the attachment of an external component that increases the sensor complexity and cost.

In this Letter we propose and demonstrate a novel (to the best of our knowledge), yet quite simple, fiber-optic level sensor based on multimode interference (MMI) effects that can be used for continuous or discrete liquid level detection. The advantage of MMI devices are that they exhibit a passband filter response, and they only require splicing a specific section of multimode fiber (MMF) between two single-mode fiber (SMF) segments [14]. In our sensor, we use an MMF known as no-core fiber, which has a diameter of $125 \mu \mathrm{m}$ without clad; i.e., the cladding is air. Therefore, when the MMF is immersed in liquid, there is a shift of the spectral response of the sensor, and the level of the liquid can be correlated with the spectral shift. The sensor also allows for multiplexed operation by simply changing the length of the MMF, which changes the peak wavelength response of the sensor. The fabrication of such sensors is straightforward and relatively cost effective, which is very promising for many applications.

A schematic of the sensor is shown in Fig. 1. The key component is a segment of the no-core $\mathrm{MM} \overline{\mathrm{M}}$ with a precise length. In our sensor, the MMF is spliced to a single segment of SMF, while the other end of the MMF is coated by evaporating a $200 \mathrm{~nm}$ gold layer to act as a mirror. When the MMF is in air, the peak wavelength response of the MMI device for the fourth self-image can be easily calculated from the following equation:

$$
\lambda_{0}=4 \frac{n_{\mathrm{MMF}} D_{\mathrm{MMF}}^{2}}{L},
$$

where $n_{\mathrm{MMF}}$ and $D_{\mathrm{MMF}}$ correspond respectively to the effective refractive index (RI) and diameter of the fundamental mode of the MMF, and $\lambda_{0}$ is the free space wavelength $[14,15]$. The fourth self-image (and multiples) is used because we obtain the narrowest spectral bandwidth and minimum insertion losses. When light at wavelength $\lambda_{0}$ is launched into the MMF from the SMF, the input field at the SMF-MMF interface will form a

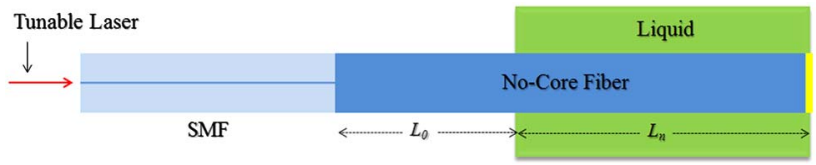

Fig. 1. (Color online) Schematic of the fiber-optic liquid level sensor. 
self-image that is an exact replica of the input field at the MMF-mirror interface. Because of the mirror, this self-image is reflected back and coupled into the SMF. Therefore, the bandpass response of the MMI device is not affected by the mirror and we can interrogate the sensor through the SMF. The advantage of this configuration is that we can do remote testing of the sensor. Additionally, as shown in Eq. (1), we can choose the initial peak wavelength of our sensor by just changing the MMF length, thus providing a very simple wavelength multiplexing operation.

When a section of the no-core MMF is surrounded by a liquid, as shown in Fig. 1, this section now has a cladding whose RI is the same of the liquid. Therefore, the RI difference between the core and cladding is reduced, and as a result of the Goos-Hänchen shift [15], the effective diameter of the fundamental mode of the no-core fiber is increased. This should also increase the effective RI, because the evanescent filed penetrates more into the liquid. According to Eq. (1), when $n_{\mathrm{MMF}}$ and $D_{\mathrm{MMF}}$ are increased, the peak wavelength should be shifted to longer wavelengths. Nevertheless, in the schematic shown in Fig. 1, the fiber is not entirely covered by the liquid, and Eq. (1) cannot be used to estimate the final peak wavelength. If we look carefully at Fig. 1 , we notice that the MMI appears to now be formed with two slightly different fibers. We have one section of no-core fiber in air, while the rest of the no-core fiber is immersed in the liquid. The resulting effect is similar to having two different MMF with slightly different effective refractive indices (RIs) and diameters. Therefore, the required phase factor is now determined by the contribution of these two MMFs. The peak wavelength shift is then estimated from Eq. (2):

$$
\lambda_{0}=\frac{4 n_{\mathrm{MMF} n} D_{\mathrm{MMF} n}^{2}}{L}\left(\frac{L_{n}}{L}\right)+\frac{4 n_{\mathrm{MMF}} D_{\mathrm{MMF}}^{2}}{L}\left(\frac{L_{0}}{L}\right),
$$

where the first part in the sum is the contribution of the segment of the no-core fiber with liquid around and the second part is the contribution of the no-core fiber without liquid. Here $L$ is the total no-core fiber length, $L_{0}$ is the no-core fiber length without liquid, $L_{n}$ is the no-core fiber length with liquid, $n_{\mathrm{MMF} n}$ and $D_{\mathrm{MMF} n}$ are the effective RI and diameter for the no-core fiber length with liquid (as calculated from the Goos-Hänchen effect). According to Eq. (2), if all the MMF is in air, then $L_{n}$ is zero and $L_{0}=L$, which gives the same equation as Eq. (1). On the other hand, if a section of the MMF is covered with liquid, we add the contribution of each segment of the MMF in air and liquid. In this way, we can easily find the final peak wavelength of the MMI sensor due to the level of liquid covering the MMF. Therefore, by placing the MMF in the vertical direction, changes in the liquid level can be easily detected by monitoring the peak wavelength shift as a function of the liquid level. We should also highlight that the sensor operates in the way described by Eq. (2), as long as the RI of the liquid is lower than the no-core MMF $(n=1.44402)$. Additionally, after estimating the parameters of the fiber (air and liquid), only the ratio of the fibers will be modified when the liquid level is changed and this provides a linear response of the sensor.

The sensor is demonstrated by splicing a section of no-core fiber to an SMF. The no-core fiber length is $60.816 \mathrm{~mm}$, providing a peak wavelength of $1484 \mathrm{~nm}$. Finally the facet of the no-core fiber is coated with $200 \mathrm{~nm}$ of gold using a thermal evaporator. The sensor is placed inside a channel formed in a block of acrylic to facilitate the changing of the level of the liquid, and it also allows the use of different liquids. The response of the liquid level sensor was tested using an Agilent tunable laser with a wavelength range from 1460 to $1580 \mathrm{~nm}$. The tunable laser was coupled into port 1 of an optical circulator, and the output from port 2 is connected either directly to the SMF of our sensor or to a $1 \times 2$ splitter $(3 \mathrm{~dB}$ splitting ratio) to allow testing of two sensors simultaneously. Light that comes back into port 2 after propagating through the sensor is detected at port 3. Light was measured using an InGaAs photodetector connected to a Keithley digital multimeter. The setup was fully controlled through GPIB ports using LabVIEW.

The response of the sensor for $10 \mathrm{~mm}$ increments of ethylene glycol is shown in Fig. 2(a). As expected, the peak wavelength is shifted to longer wavelengths as
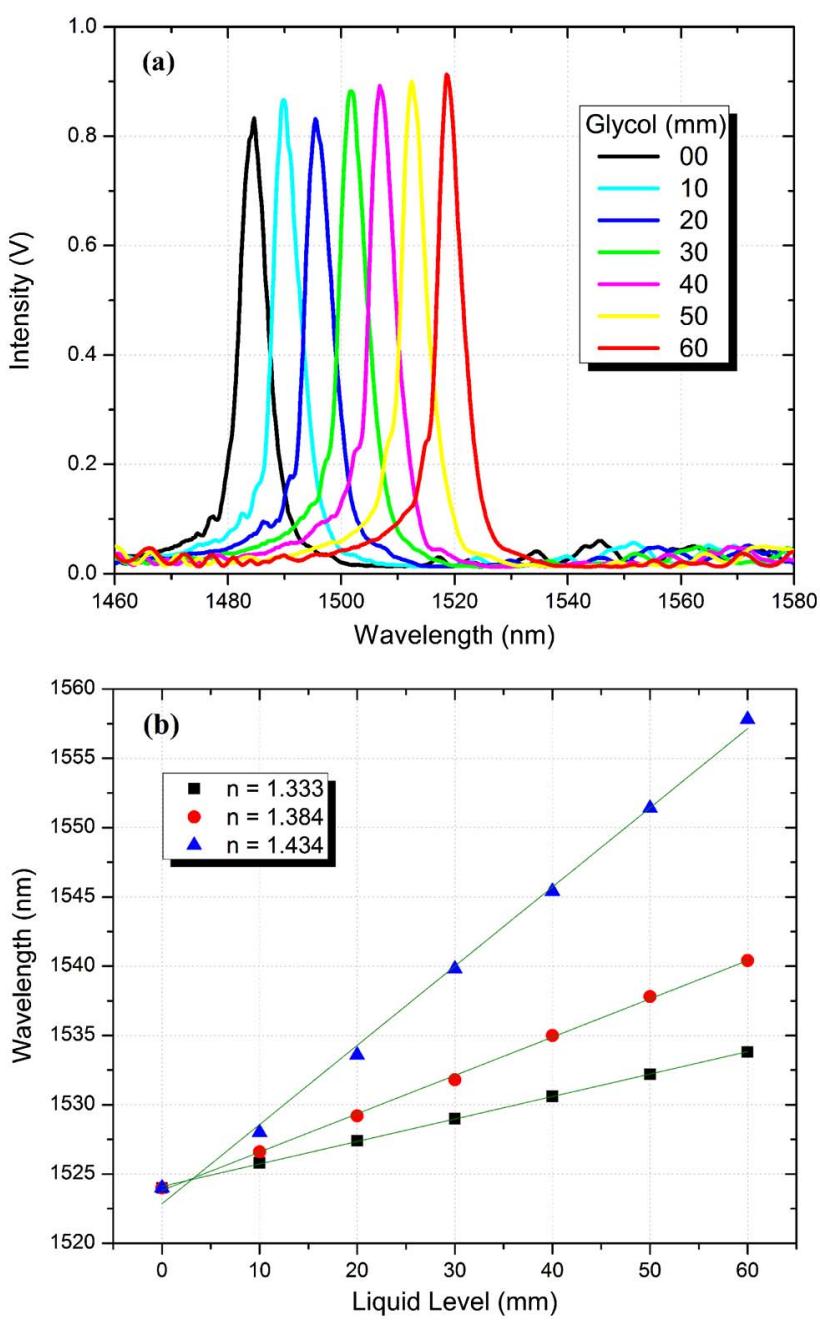

Fig. 2. (Color online) (a) Sensor response for ethylene glycol operating at a peak wavelength of $1484 \mathrm{~nm}$ and (b) liquid level sensing for liquids with different refractive indices. 

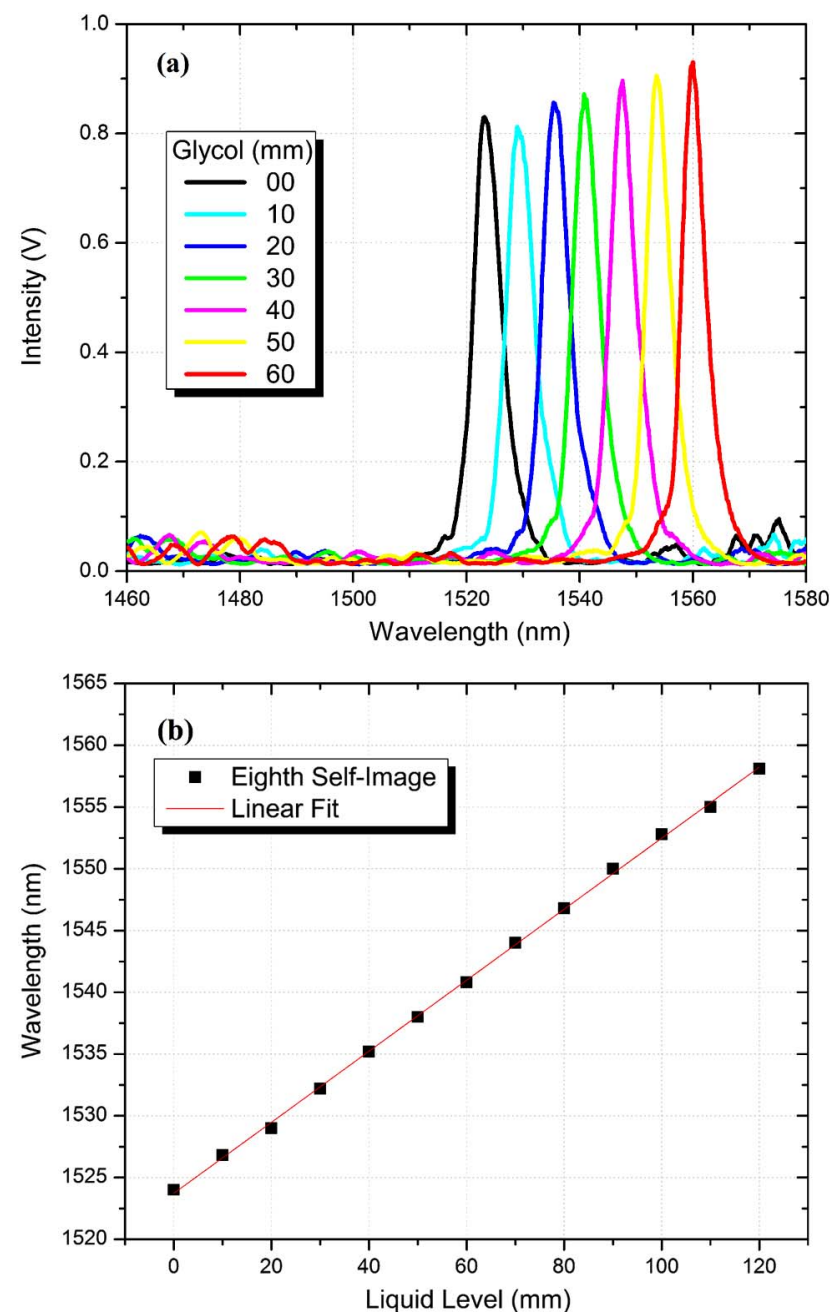

Fig. 3. (Color online) (a) Sensor response for ethylene glycol operating at a peak wavelength of $1524 \mathrm{~nm}$ and (b) sensor response for ethylene glycol operating at the eighth self-image.

the liquid level is increased and has a linear response, as shown in the blue triangles of Fig. 2(b). The slight deviation from linearity at $10 \mathrm{~mm}$ is due to the sensitivity of the sensor if the liquid is not increased exactly by $10 \mathrm{~mm}$. Also shown here are the results for two different liquids with different RIs $n=1.333$ and $n=1.384$. We note that they exhibit a similar response, but the magnitude of the wavelength shift is reduced. This is due to the fact that the accumulated phase of the modes is less for a lower RI. This provides another advantage to our sensor, because we can also determine what kind of liquid we are handling based on the RI value.

The sensor can easily be multiplexed by simply cleaving a no-core fiber with a different length, which provides a different peak wavelength. As shown in Fig. 3(a), a sensor with a peak wavelength of $1524 \mathrm{~nm}$ exhibits very similar response when the level of ethylene glycol is modified, but operating with a different wavelength range. This allows a very simple, yet very robust multiplexed liquid level system. Another important advantage of our sensor is that the sensing range can be increased by using a longer section of no-core fiber and operating the MMI region at a higher self-image order (exact multiple of the fourth image). Therefore, by using a no-core fiber length of $121.632 \mathrm{~mm}$, we can operate at the eighth self-image and the peak wavelength does not change as compared to the fourth self-image. Nevertheless, when the sensor is tested using ethylene glycol, the same wavelength shift as in the case of the fourth image, but now we can detect a liquid level of almost $120 \mathrm{~mm}$. This could be expanded to higher self-image orders, thus providing a simple way to increase the sensing range. If discrete operation is required, we could cover the upper $40 \mathrm{~mm}$ of the no-core fiber, either with a low RI material, such as polydimethylsiloxane, or by preventing the liquid from reaching that section and having the liquid interact only within the first $20 \mathrm{~mm}$ of the no-core fiber. In this situation, the peak wavelength will shift between 3.4 and $11 \mathrm{~nm}$ based on the RI of the liquid, which is enough to detect when the liquid makes contact with the fiber. The attenuation ranges from 5.2 to $13 \mathrm{~dB}$, measured at the initial peak wavelength, when going from water to ethylene glycol, which is low enough for discrete liquid level sensing.

In summary, we have proposed and experimentally demonstrated a very simple and highly functional liquid level sensor based on MMI effects. The sensor provides an excellent linear response, with the ability to discriminate the RI of the liquid. Both the multiplexing operation and sensing range can be easily achieved by just controlling the length of the no-core fiber. This kind of sensor is very promising not only for continuous or discrete liquid level sensing, but also for RI sensing applications because they can easily discriminate the RI of the liquid.

\section{References}

1. B. W. Northway, N. H. Hancock, and T. Tran-Cong, Meas. Sci. Technol. 6, 85 (1995).

2. F. N. Toth, G. C. M. Meijer, and M. van-der-Lee, IEEE Trans. Instrum. Meas. 46, 644 (1997).

3. A. Wang, M. F. Gunber, K. A. Murphy, and R. O. Claus, Sens. Actuators A 35, 161 (1992).

4. P. Raatikainen, I. Kassamakov, R. Kakanakov, and M. Luukkala, Sens. Actuators A 58, 93 (1997).

5. E. Udd, Fiber Optic Sensors: An Introduction for Engineers and Scientists (Wiley, 2006).

6. K. Iwamoto and I. Kamata, Appl. Opt. 31, 51 (1992).

7. F. Perez-Ocon, M. Rubino, J. M. Abril, P. Casanova, and J. A. Martinez, Sens. Actuators A 125, 124 (2006).

8. S. Khaliq, S. W. James, and R. P. Tatam, Opt. Lett. 26, 1224 (2001).

9. M. Lomer, A. Quintela, M. Lopez-Amo, J. Zubia, and J. M. Lopez-Higuera, Meas. Sci. Technol. 18, 2261 (2007).

10. S. M. Chandani and N. A. F. Jaeger, Opt. Eng. 46, 114401 (2007).

11. P. Nath, P. Datta, and K. Ch. Sarma, Microw. Opt. Technol. Lett. 50, 1982 (2008).

12. M. Bottacini, N. Burani, M. Foroni, F. Poli, and S. Selleri, Microw. Opt. Technol. Lett. 46, 520 (2005).

13. I. K. Ilev and R. W. Waynant, Rev. Sci. Instrum. 70, 2551 (1999).

14. J. E. Antonio-Lopez, A. Castillo-Guzman, D. A. May-Arrioja, R. Selvas-Aguilar, and P. LiKamWa, Opt. Lett. 35, 324 (2010).

15. L. B. Soldano and E. C. M. Pennings, J. Lightwave Technol. 13, 615 (1995). 